Pesq. Vet. Bras. 35(10):842-843, outubro 2015

DOI: $10.1590 / \mathrm{S} 0100-736 \mathrm{X} 2015001000004$

Topic of General Interest

\title{
Nanotechnology: meeting the future of Veterinary Parasitology Research ${ }^{1}$
}

\author{
Amilcar Arenal Cruz ${ }^{2 *}$ and Marcelo Beltrão Molento ${ }^{3,4 * *}$
}

\begin{abstract}
Cruz A.A. \& Molento M.B. 2015. Nanotechnology: meeting the future of Veterinary Parasitology Research. Pesquisa Veterinária Brasileira 35(10):842-843. Laboratório de Doenças Parasitárias, Setor de Ciências Agrárias, Universidade Federal do Paraná, Rua dos Funcionários 1540, Curitiba, PR 80035-050, Brazil. E-mail: molento@ufpr.br

The field of nanotechnology involves an array of different areas of expertize with the application of innovative products in Medicine, Engineering, and to a less extent to Veterinary Medicine. In our opinion, more research is needed, in special to Animal Parasitology, to develop state of the art products to solve old problems. Livestock, pets and wildlife may benefit from products in nanoscale, such as vaccines, target recombinant proteins, or new drug candidates. Thus, we want to give some food for thought to drive scientific programs into nanotechnology, creating a safer environment to animals and humans.
\end{abstract}

INDEX TERMS: Biotechnology, parasite control, vaccines, RNAi.

NANOTECHNOLOGY literally means any technology performed on a nanoscale that has practical applications to our everyday activities. This encompasses the production and usage of physical, chemical, or biological systems at scales ranging from individual atoms or molecules to submicron dimensions, as well as the integration of the resulting nanostructures into larger systems (Bhushan 2010).

The Fantastic Voyage (1966) was the first movie that illustrated the use of nanotechnology in medicine to the large public. In it, the scientist Jan Benes was saved from a blood clot by an expedition using the submarine Proteus, miniaturized to the scale of $1000 \mathrm{~nm}$. For this impressive screenplay, the film won the Oscar for best special effects. The brilliant creativity of the writer of the film, Harry Kleiner, was only the beginning of the nanomedicine breakthrough of the following three decades.

More recently, the funding for nanotechnology research was in the order of US\$67 billions, running at US $\$ 10$ billion/year (Cientifica 2013), mainly in the USA, Germany, China and Japan. By 2013, the USA multi-agency National

\footnotetext{
${ }^{1}$ Received on April 30, 2015.

Accepted for publication on August 7, 2015.

${ }^{2}$ Laboratório de Bioquímica, Faculdade de Ciências Veterinárias, Universidade de Camagüey, Camagüey, Cuba. *Recipient of CAPES/PVE-2012, as Visiting Professor.

${ }^{3}$ Laboratório de Doenças Parasitárias, Setor de Ciências Agrárias, Universidade Federal do Paraná (UFPR), Rua dos Funcionários 1540, Curitiba, PR 80035-050, Brazil. **Corresponding author: molento@ufpr.br

${ }^{4}$ Instituto Nacional de Ciência e Tecnologia (INCT-Pecuária), Av. Antônio Carlos 6627, Belo Horizonte, MG 31270-901, Brazil.
}

Nanotechnology Initiative, has dedicated nearly US $\$ 1.8$ billion to nanotechnology, where US $\$ 408$ millions was related to life sciences (Nano 2013). The European Union countries have a new financial instrument called Horizon 2020, which came to replace the successful Seventh Framework Program initiative by the end of 2013. Running from 2014 to 2020 , the proposed EU $\$ 80$ billion research money aims to harness innovation to drive new growth and jobs in the region (European Commission 2013). Since 2011, China's nanotech funding has already surpassed the USA centering the resources into drug delivery and medical diagnostic research (Cientifica 2013).

Nanotechnology has given the tools to medicine to improve treatments and to develop effective immune responses against infectious agents. Cancer research has been the major area met by nanotechnology. Diseases caused by bacteria, fungi and viruses have also been targets for nanoparticles. Nanotech can offer an improvement in imaging and diagnosis of the diseases as well, slipping away the limitations of biological barriers. Despite the advances of the nanomedicine, the technology is still a long way from perfection due to the difficulties to get the milestone of clinical trials, where only a few nanoproducts have arrived to the clinical phase (Bourzac 2012).

The French Veterinary Academy held the first conference on nanotechnology in veterinary medicine this year in Jouy-en-Josas, presenting ground breaking research on the subject, identifying promising areas to pursue and discuss the foreseeable impact on veterinary pharmacology and diagnostic capabilities. Even with the fact of the advance- 
ment of nanotech to human health, parasitic diseases have only a few applications and the reports related to nano-parasitology focus mostly on Leishmania sp. and Plasmodium sp. (Carvalho et al. 2013, Waknine-Grinberg et al. 2013). But they have demonstrated the potentialities of nanotech applications into research and development.

The development of a new drug delivery system favoring longer dose intervals and the increase of drug activity against parasites are some of the major issues studied in nano-parasitology. Active and non-active targeting has been explored using liposomes, solid lipid nanoparticles and hydrophobic polymeric nanoparticles (Santos-Magalhães \& Mosqueira 2010). Nanoparticles carriers have also improved the bioavailability and drug selectivity, even in very complicated treatments like cerebral malaria by Plasmodium falciparum infection (Waknine-Grinberg et al. 2013).

One of the most important problems in parasitology research as well as, to the Veterinary counterpart is the development of drug resistance (Molento 2009). Nanoparticles may offer a potent tool to reduce the risk of resistance to traditional drugs, avoiding some of the resistance mechanisms, increasing drug bioavailability and potentiating the target of the treatment. New antiparasitic drugs can also be developed with nanotechnology like the case of Tio2@ Ag nanoparticles (Allahverdiyev et al. 2013), being an eco-friendly and inexpensive approach for use in veterinary medicine.

Pegylated or polyplex nanoparticles offer fantastic options to increase the biodistribution of a compound. Nanoparticles can help veterinary medicine in mass treatments of ectoparasites, facilitating the drug-parasite interaction. The effectiveness of phytotherapeutic products can be improved, like the recent treatment of antiparasitic silver nanoparticles using Cissus quadrangularis against Hippobosca aculata and Rhipicephalus (Boophilus) microplus (Santhoshkumar et al. 2012). It could also allow the potential recombinant proteins to act at different drug binding sites, as nanovectors (Devalapally et al. 2007). The active gold coated glass particles commonly known as nanoshells could help veterinary medicine to improve the treatment of parasites facilitating the target of the parasite and the drug delivery, physically destroying the infection. Also nanoshells can be useful in the study of host-parasite as biosensors. Nanodevices can allow the diagnostic of parasite in herds allowing veterinarians to suggest a correct health management. We would like to let you know that our research groups in Cuba and Brazil are currently running preliminary test using nanopeptides against ruminant helminths (Haemonchus contortus and Fasciola hepatica).

Nanotechnology can also allow the development of adjuvants for veterinary parasite vaccines, boosting their present low efficacy. Vaccines using nanoparticles as adjuvants have the further potential to induce both antibody and cellular immune response by simultaneously activating the major histocompatibility complex class I and class II pathways or increase the antigen visibility and their lifespan, to meet the host immune system. Recently, a nano-microparticulated malaria vaccine elicited long-lasting protec- tive antibody titers with only a single dose (Dinglasan et al. 2013). Although it is necessary that quantum chemistry overcome their limitations, new nanodevices could allow a better comprehension of the host-parasite interaction. It can also help to facilitate the studies of parasites life cycle and their geographical distribution. Thus, nanoparticles are a strong tool to elucidate the parasite gene function in the era of omics.

\section{CONCLUDING REMARKS}

Despite the potentialities of the use of nanotechnology in veterinary parasitology research we think that only a few successful applications have been accomplished so far. A small number of parasites have been the target for nanotechnology, focusing primarily in Leishmania sp. and Plasmodium sp. research. Although various benefits were demonstrated, a more active research is necessary to apply this technology to the real needs of animal health, taking into account the economic constraints of this sector. Nanotechnology as any other technology is getting good exposure in Brazil, but researchers must also take into account the ethical and regulatory issues (scientific monitoring, risk assessment, target species, aberrant results and safety to other organisms) before its widespread use.

\section{REFERENCES}

Allahverdiyev A.M., Abamor E.S., Bagirova M., Baydar S., Ates S. \& Kaya F. 2013. Investigation of antileishmanial activities of Tio2@Ag nanoparticles on biological properties of L. tropica and L. infantum parasites in vitro. Exp. Parasitol. 135:55-63.

Bhushan B. 2010. Introduction to Nanotechnology, Springer Handbook of Nanotechnology. 3rd ed. Springer, Heidelberg, Germany. 15p.

Bourzac K. 2012. Nanotechnology: carrying drugs. Nature 491:S58-60.

Carvalho R.F., Ribeiro I.F., Miranda-Vilela A.L., Souza Filho J., Martins O.P. \& Silva D.O. 2013. Leishmanicidal activity of amphotericin B encapsulated in PLGA-DMSA nanoparticles to treat cutaneous leishmaniasis in C57BL/6 mice. Exp. Parasitol. 135:217-222. Cientifica 2013. Global Funding of Nanotechnologies. 2011 Edition. <http://www.cientifica.com/ research/market-reports/nanotech- funding-2011>

Devalapally H., Chakilam A. \& Amiji M.M. 2007. Role of nanotechnology in pharmaceutical product development. J. Pharm. Sci. 96:2547-2565.

Dinglasan R.R., Armistead J.S., Nyland J.F., Jiang X. \& Mao HQ. 2013. Single-dose microparticle delivery of a malaria transmission-blocking vaccine elicits a long-lasting functional antibody response. Curr. Mol. Med. 13:479-487.

European Commission 2013. Breakdown of the "Horizon 2020" budget. <http://ec.europa.eu/research/ horizon2020> Access on Dec. 9, 2013.

Molento M.B. 2009. Parasite control in the age of drug resistance. Vet. Parasitol. 3:188-196.

Nano 2013. Budget brief for 2013. National Nanotechnology Initiative. Online Dec. 9, 2013 <http://www.nano. gov/node/750>

Santhoshkumar T., Rahuman A.A., Bagavan A., Marimuthu S., Jayaseelan C. \& Kirthi A.V. 2012. Evaluation of stem aqueous extract and synthesized silver nanoparticles using Cissus quadrangularis against Hippobosca maculata and Rhipicephalus (Boophilus) microplus. Exp. Parasitol. 132:156-165.

Santos-Magalhães N.S. \& Mosqueira V.C.S. 2010. Nanotechnology applied to the treatment of malaria. Adv. Drug Del. Rev. 62:560-575.

Waknine-Grinberg J.H., Even-Chen S., Avichzer J., Turjeman K., BenturaMarciano A. \& Haynes R. 2013. Glucocorticosteroids in nano-sterically stabilized liposomes are efficacious for elimination of the acute symptoms of experimental cerebral malaria. PLoS ONE 8:2722. 